\title{
ASKfm: Motives of Self-Disclosure to Anonymous Questions
}

\author{
Pheseline Felim¹, Dindin Dimyati², Mohammad Shihab3 \\ 1President University, Email: im_pheseline@hotmail.com \\ 2President University, Email: dindin.dimyati@president.ac.id \\ 3President University, Email: shihab@president.ac.id
}

\begin{abstract}
Self-disclosure may happen greatly in social media because nonverbal cues were reduced and their anonymity feature, while they also control their content distribution to certain boundaries based on their own privacy management. The purposes of this research are to know motivation of ASKfm users to disclose their personal information to anonymous questions and how privacy rule criteria such as gender, culture, and context make them want to disclose their personal information to anonymous questions. By using in-depth interview, data were collected from three males and three females aged 18 to 24 years old. They had ASKfm accounts, had answered anonymous questions at least five times, had minimum 100 likes in total, and had revealed personal information to the anonymous questions. ASKfm involved personal evaluation towards rewards and costs received by the users. Participants were motivated to disclose their personal information to anonymous questions to get enjoyment by receiving and answering many anonymous questions as well as relationship building. The motivations indirectly caused self-presentation in which some participants promoted their ASKfm accounts in other social media to get more questions. Collectivistic participants received benefit on enjoyment, while individualistic participants who received benefit on relationship building and maintenance. Participants were more careful in managing disclosures because they were aware of possible risks and possibility of widely personal information distribution to unknown publics by filtering the questions rather than changing the Privacy Settings.
\end{abstract}

KEYWORDS: anonymity, anonymous questions, ASKfm, motivations, privacy management, self-disclosure 


\section{INTRODUCTION}

Social media enable users to have communication without showing physical body language and physical appearance as happens in face-to-face communication (McKenna et al., 2002). Anonimity feature of social media also makes users now have access to powerful multimedia tools to decide their desirable identity - publicly known or totally private - when having communication with other users to overcome their fear of disapproval and sanctions by those in their real-life social circles (Bargh et al., 2002).This may be the cause why self-disclosure in online social networking happens greatly, especially for shy and lonely users, to develop interpersonal relationship.

Social media provides privacy controls or privacy settings that allow users to have power over their posts (time, pace, contents) including personal information, so fear of possible risks is reduced (Tian, 2013). Consequently, some users are fine to share their personal information, while other user will limit their post to a very small and carefully controlled group (Golbeck, 2015, p. 31). It means that each user has their own concerns, motivations, and privacy management to what extent he/she reveals personal information and to whom in online social networking which depend on factors influencing selfdisclosure (for review Krasnova, Kolesnikova, \& Guenther, 2009; Veltri, Krasnova, Elgarah, 2011; Waters \& Ackerman, 2011; Tian, 2013; Masur \& Scharkow 2016; Al-Kandari, Melkote, \& Sharif, 2016; Liang, Shen, \& Fu, 2016).

There have been many researchers explaining the motivation of online selfdisclosure. However, most of them focused on psychological and interpersonal needs (e.g Tian, 2013; Chen, 2015; Lai \& Yang, 2015) or privacy concerns (e.g. Christofides, Muise, \& Desmarais, 2012;
Tsay-Vogel, Shanahan, \& Signorielli, 2016; Masur \& Scharkow, 2016) on Facebook, Twitter, and Blogs. Chen (2015) and Lai and Yang (2015) argued that social media function is for "social", but they will turn to other functions because of different services or features and privacy policy to fulfill specific needs of the users. Thus, there is a suggestion from previous researchers to take focus on different features use instead of taking overall perspective because "social consequences of the Internet may partly depend on individual's motives for using medium" (Tian, 2013).

This research filled the gap by taking focus on why ASKfm users disclose their personal information to anonymous questions based on their evaluation towards gaining rewards and costs as well as gender, culture, and context. There have been so far just few researches on ASKfm. Their focus is on cyberbullying (e.g. Han \& $\mathrm{Lv}, 2014$ ) or strategic anonymity in the social lives of adolescents (e.g. Ellison, Blackwell, Lampe, Trieu, 2016). Therefore, this research is significant to add insight about a specific social media feature for better and complete understanding on online self-disclosure in Q\&A-style and semi-anonymous social media.

\section{LITERATURE REVIEW}

\subsection{Motivation of self-disclosure in social media}

Online self-disclosure includes the activity of sharing views, opinions, and personalities (Al-Kandari et al., 2016) through visual form and verbal description such as updating profile information, uploading photos and videos, tagging photos, posting status updates, posting comments, and communicate news or text message on an social network site (SNS) walls (Tsay-Vogel et al., 2016; Krasnova. et al., 2010, p. 382; Rui \& Stefanone, 2013b, 
p. 1287, as cited in Ahmed, 2015) about personally identifiable information and offline activities (Humphreys et al., 2014). Personal information are various, for instance, attitudes toward other religions, nationalities, races, financial status, how much money they earn, how much they owe, how much they have saved, feelings about parents, sexual fantasies, physical and mental health, ideal romantic partner, drinking and/or drug behavior, the most embarrassing moment, unfulfilled desires, and self-concept (DeVito, 2014, p. 56).

Researches on self-disclosure in social media show that users' selfdisclosures are the result of their personal evaluation towards gaining of rewards or expected benefits (e.g. Krasnova et al., 2009; Veltri et al., 2011; Waters \& Ackerman, 2011; Al-Kandari et al., 2016; Chen, 2015; Lai \&Yang, 2015; Bevan-Dye \& Akpoviji, 2016; DeVito, 2014, p. 58) and costs or privacy concerns (e.g. Krasnova et al., 2009; Veltri et al., 2011; DeVito, 2014, p. 58; Tian 2013; Liang et al., 2016; Masur \& Scharkow, 2016). Turbulence between expected benefits and privacy risk is likely to occur in social media (Trepte, 2015). This situation supports the core idea of social exchange theory by Thibaut and Kelley:

"People will calculate the costs and rewards of a given situation and guide their behaviors accordingly .... Costs are those elements in the relationship that have negative value to a person (e.g. stress, time, energy, attention). Rewards are those elements in the relationship that have positive value to a person (e.g. fun, loyalty, attention)" (as cited in West \& Turner, 2010, p. 186).

Rewards refer to some forms of benefits gained by users. First, enjoyment which is experienced by users because of the features provided by social media
(Krasnova et al., 2009; Veltri et al, 2011). Waters \& Ackerman (2011) argued that Facebook users share private information in online conversation or updating status for improving psychological well-being such as fun, enjoyable activity, and relieving stress. Facebook users (Drussell, 2012, as cited in Sheldon, 2015, p. 9) and Instagram users (Araújo, Corrêa, da Silva, Prates, \& Meira, 2014) enjoy to receive "likes" or responses on their post that is keeping them to upload attractive posts to catch more attention. Those who disclose their personal information may receive responses such affirmation, support, and engagement (Chen, 2015; Barack \& GluckOfri, 2007, p. 408; Khan \& Antonnuci, 1980; House, 1986, as cited in Ahmed, 2015) which might not be received in faceto-face communication.

Second, research conducted by Krasnova et al. (2009) shows that Facebook and MySpace allow users to participate and (self-) communicate, hence enable them to have efficient communication with large group of social media members at the same time by posting one status. Consequently, there is possibility for reciprocation of information (Krasnova et al., 2010, as cited in Sheldon, 2015, p. 10; Waters \& Ackerman, 2011) that leads to both relationship building and relationship maintenance between people who trust, respect, and care each other (DeVito, 2014, p. 58; Waters \& Ackerman, 2011). Research of Tian (2013) stated that bloggers with higher social anxiety are able to make new friends and pursue to close relationship (good quality of relationship). This is because blogs provide anonymity feature, lack of social cues, and greater control over their online communication. When bloggers and the readers who have already known each other in offline or non-virtual world, they also disclosed more about themselves in the blogs to maintain the relationship. 
Third, social media users disclose their personal information for selfrepresentation (Veltri et al., 2011; Christofides et al., 2009, p. 343). According to Goffman (1959, as cited in Sheldon, 2015, p. 37), self-presentation is one's unique roles in different situation. In social media context, self-presentation can be related to the need of popularity among other network members which may be the strongest factor for social media users to have self-disclosure (Lai \& Yang, 2015). For example, research of Waters \& Ackerman (2011) shows that Facebook users want to show off their popularity and want to be perceived as person who always keep up with the trends, hence they post events of their experience to others.

Costs refer to privacy concerns individual assessment about what happens to one's information once it is disclosed. Previous studies argued that privacy concerns will lead to active privacy protection and self-disclosure (Stutzman, Capra, \& Thompson, 2011, as cited in Liang et al., 2016). The assessment is reflected by degree of privacy concerns which are determined by the perceived likelihood of a privacy violation and perceived damage of the social media (Krasnova et al., 2009 \& Veltri et al., 2011), as well as trust in social media members and legal assurance (Veltri et al., 2011).

\section{Perceived likelihood of a} privacy violation is the possible occurrence of various violation and negative event in social media (Krasnova et al., 2009; Veltri et al., 2011). Social media users have been already aware about the possibility of the privacy violation as they have been reminded through the Privacy and Terms section in the social media. However, this perceived likelihood of a privacy violation is usually distorted by "optimistic bias" attitude; tendency of individuals to see a negative event as less likely occurs but positive events will happen to them (Krasnova et al., 2009). This attitude causes the social media users tend to ignore the legal consequences stated in the social media privacy policy because they think that third parties have little interest in their data and consider their information is trivial. Moreover, Madden, M. (2012) also stated that when social media users never get negative experience, they will decrease their concerns about sharing information, so they are willing to share all personal details (physical location, photos of their children, etc.). Although privacy is important to social media users, nevertheless their actions show otherwise result. For example, Rainie and Madden (2015) reported that only $30 \%$ of all adults in U.S. have taken steps to protect their privacy online such as changing their privacy settings on social media (as cited in Liang et al., 2016).

Perceived damage is the individual assessment of the magnitude of a negative event and its consequences (Krasnova et al., 2009; Veltri et al., 2011). Some damages in social media are financial loss, credit damage, unsought e-mails or phone calls, identity theft, negative psychological and social consequences like detrimental impact on one's sense of worth, social standing and relations (Krasnova et al., 2009; Liang et al., 2016). Telling more personal information cause (1) the higher possibility of other people (receivers) to use that information to against the discloser (personal risk), (2) problems such as rejection, deterioration of relationship, or even termination of relationship (relationship risk), and (3) losses in professional world, for instance, disclosing about consuming drugs and alcohol in workplace may cause dismissal, demotion, or social exclusion (professional risk) (DeVito, 2014, p. 58; Tian, 2013). Moreover, Krasnova et al. (2009) argued that although perceived damages influence users' privacy concerns, they are not as 
significant as perceived likelihood of privacy violation.

Trust in social media members and legal assurance also plays important role when people choose to share their personal information (Berger and Calabrese, 1975, as cited in Sheldon, 2015, p. 6; DeVito, 2014, p. 57). They tend to disclose to someone whose behaviors have been known and trusted by them. When the levels of trust increases, online disclosure will follow accordingly (Masur \& Scharkow, 2016). Thus, those who disclose more in social media are perceived as more trustworthy and are more likely to elicit disclosure from other users (Henderon \& Giding, 2004, as cited in Christofides et al., 2012). In contrast, people with less disclosure in social media will limit accessibility of their information by preserving their face, distinct boundary between friends and strangers (Veltri, Krasnova, \& Elgarah, 2011).

Finally, social media users' motivation to get benefits and their privacy concerns in social media determine the degree of online disclosure; the more benefits gained through the use of social media, the more people will disclose about themselves, and the higher privacy concerns that the users possess, the less online self-disclosure occurs because they are aware that their personal information may be used irresponsibly (Krasnova et al., 2009; Veltri et al., 2011; Bevan-Dye \& Akpoviji, 2016; Walther, 2011, as cited in Masur \& Scharkow, 2016), which may cause them to not imply sophisticated privacy settings.

\subsection{Communication privacy management in social media}

The evaluation towards rewards and costs in online self-disclosure vary from one user to other users because of some privacy rule criteria, such as (1) gender (differences between men and women in managing privacy boundaries), (2) culture (norms of privacy and openness in a given culture), and (3) context ("social environment" and "physical setting" that determines people develop rules for privacy or disclosure) (Petronio, 2002, as cited in West \& turner, 2010, p. $223 \&$ 229-230). These criteria are mutually agreed upon, constructed, established, and managed from one's ownership into collective rule boundaries. To conclude, privacy management is "selective boundary control which is enacted in multiple behavioral mechanism and strategies." (Masur \& Scharkow, 2016).

Gendered criteria affect to how men and women disclose their personal information. It is believed that women have more self-disclosures than men on the web (Punyanunt-Carter, 2006, as cited in Chen, 2015). Women will increase the depth of their self-disclosure as the relationship become more intimate, while men stay at the same level (Goodwin \& Lee, 1994, as cited in DeVito, 2014, pg 57). It is because women have less taboo topics information that will not be disclosed to their friends - than men have (Goodwin \& Lee, 1994, as cited in DeVito, 2014, p. 57). However in America and Argentina, men have more willingness to self-disclose than women do (Horenstein \& Downey, 2003, as cited in DeVito, 2014, p. 57). This happened greatly in initial relationship; men will disclose more than women to "control the relationship's development" (Derlega, Winstead, Wong, \& Hunter, 1985, as cited in DeVito, 2014). Similarly, women tend to be more concerned about privacy than men (Fogel \& Nehmad, 2009, as cited in Christofides, Muise, and Desmarais, 2012), hence they are more careful when disclose their personal information. 
This research took cultural dimensions of Hofstede such as collectivistic and individualistic cultures for the cultural criteria which influence the users to disclose their personal information. Collectivistic societies (e.g. societies in Pakistan, Colombia, Venezuela, Peru, much of African and Asian countries such Indonesia) who value more on harmonious relationships of in-group rather than oneself (Samovar, Porter, \& McDaniel, 2009, p. 198, 200-201; Novera, 2004). On the other side, individualistic societies (e.g. societies in United States, Australia, Great Britain, Canada, the Netherlands, and New Zealand) value more on personal rights, self-expression, and achievement rather than loyalty in a group (Samovar, Porter, \& McDaniel, 2009, p. 199-200). Crosscultural research of Liang et al. (2016) toward Twitter users shows that collectivistic societies tend to have more effective privacy settings in doing self-disclosure than individualistic societies. Collectivistic societies perceive in-group and out-group differences to be larger (individualistic societies have wider boundaries than collectivistic societies) and have lower level of trust in the provider (social media), fellow social media members. They also perceive the existing laws as powerless legal assurance in protecting their privacy. Veltri et al. (2011) also indicated that Moroccans who are more collectivistic perceive Facebook are more useful in developing relationship as well as relationship maintenance since they are collectivist and need to take time to cultivate them. Meanwhile, Americans, who are more individualistic, receive more benefit from self-presentation on Facebook by publishing their life, thoughts, and experiences on Facebook. Moreover, the research also shows that individualistic country (United States) is aware that there are possibilities of negative outcomes from online disclosure, but it reflects this "optimistic bias" attitude because they believe on legal protection of privacy in their country. However, this attitude is not shown in collectivist country (Morocco) as they are aware about the likelihood of privacy violation, hence afraid of disclosing too much in social media.

In addition to cultural criteria, this research also used culture orientations of Hall which are high-context and lowcontext cultures. In high-context culture (American, Indian, Latin American, Japanese, Arab, Chinese, African American, Korean, and Indonesian), people have vague, indirect, and implicit mode of communication; they rely more on how something is said rather than what is said (Samovar, Porter, \& McDaniel, 2009, p. 215-217; Mulyana, 2008, p. 328-329). They are less open and consider conflict harmful to most communication encounters, hence meaning is conveyed through status (e.g. age, sex, education, title, etc.) and through an individual's informal friends and associates (Samovar, Porter, \& McDaniel, 2009, p. 217). For example, according to Arab Social Media Report (2005, p. 2), Arabians which have high-context culture perceive social media as tools which have positive effect in increasing quality of life of individuals. However, Arab users showed lack of trust in social media as they consider social media will cause negative effects toward social culture and tradition (as cited in Ahmed 2015). Whereas, low-context culture (e.g. German, Swiss, Scandinavian, and North American) shows the opposite; they have direct and explicit mode of communication, hence communicate more, speak more rapidly, and often raise their voices (Samovar, Porter, \& McDaniel, 2009, p. 217).

Contextual criteria such as platform used and its communication situation determines the levels of selfdisclosure. People disclose more in online 
communication such as in e-mail or social media than in face-to-face communication. It is because anonymity and invisibility offered by online communication (Suler, 2004, as cited in DeVito 2014). Research of Liang et al. (2016) on Twitter shows that women use social media generally for recreation, however, they use Facebook for engagement (social use), and use Twitter as medium for information exchange. Besides, having more followers indicates higher probability of self-disclosure because users are motivated to self-disclose for building social capital (Choi \& Bazarova, 2015) and for increasing the possibility of being retweeted (Bakshy et al., 2012; Suh et al., 2010) (as cited in Liang et al., 2016). People are more careful in managing disclosures in one-to-many context such status updates, hence use of privacy settings is important for them. Lastly, self-disclosure happens more in small groups (especially groups of two people) than in large group. It is because with one receiver, ones can monitor easily whether the receiver gives desire feedback (support) or not, whereas it is difficult to monitor large-group members because of their various responses (DeVito, 2014, p. 57).

\subsection{ASKfm}

ASKfm is a semi-anonymous Q\&Astyle social media, founded in June 16 , 2010, which is highly popular social media with over 60 million downloads of the mobile app (ASKfm, 2016) and 125 million registered users (Golbeck, 2015, p. 181). The identity of ASKfm users who post questions or comments to a profile is typically anonymous, whereas the identity of the target user is publicly known (Hosseinmardi et al., 2014). People can search ASKfm users via their name, id or email address. Anyone (even people without an account) may post on other user's profile with limitation up to 300 words.
There are some policies specific to ASKfm. First, only the target user may post an answer to a question. After answering a question, the question and its answer appear on his/her profile. Second, a user may choose to "like" at the granularity of a question-and-answer pair, but cannot like the question nor the answer individually. Liking is non-anonymous, so that the identity of the likers is publicly known. Third, ASKfm users may follow other users, but this relationship data is not available publicly and only the profile owner knows who he/she is following. Even the user who is being followed can only know how many followers he/she has and the number is not publicly visible to other users, not who is following him/her. Other users may post questions or comments on a target user's profile, and that the target user may respond each question/comment. Fourth, users are allowed to delete their posts. Fifth, users are allowed to block the users (anonymous or not) they think are very annoying, so the blocked users will be never allowed to ask users again. Sixth, people (with or without ASKfm account) can report other users' post if they feel that the post is: bullying and harassment, nude or sexually explicit content, violence and criminal activity, hate speech, self-harm, or "I just don't want to see this". The reported posts may cause the owner to be reported to police.

All contents which are posted on ASKfm including user ID, personal information or short bio (if any, as it is optional; limited to people who also have ASKfm account), total number of answers, total number of likes, total number of gifts, content of answered questions posted on a user's page, and user ID of people who liked the questions/answers can be seen by the public. All users and visitors of the site can view the posts, and the posts can be searched for in search engines, such as Google and Bing. Users' post can be also 
shared to Facebook, Twitter, and VK or into a form of link by themselves or other users with mobile devices. Moreover, trending questions and answers are showed in "Discover" page beside the "Wall" page, it means that the posts may be known more widely.

ASKfm users are protected by ASKfm's Safety Centre (partnership with some legal institutions and foundations) which provide Privacy Policy and Cookie Policy to understand the safety system of ASKfm. It also offers features which stimulate the users to share their experience and disclose personal information even to completely strangers who ask or comment on the users' profile. This is made clear by the premise of ASKfm on its website:

"We believe questions and answers are the building blocks of conversation, self-expression and deeper understanding. As the only global social network built on a Q\&A format, we are a bit different from other services you might use. How you engage with friends and express yourself on ASKfm is done through the lens of what your social connections are curious to learn about you. Through the natural exchange of questions and answers, ASKfm aims to be a safe, fun and engaging place for learning more about yourself and exploring the social world around you." (ASKfm, 2016)

ASKfm was chosen as the main focus of social media because (1) there are still few studies on ASKfm with cyberbullying topic (e.g. Hosseinmardi et al., 2014; Li, H. H. S. Et al., 2014; Hosseinmardi et al., 2015) or strategic anonymity of ASKfm users who gave questions or comment to other users (e.g. Ellison et al., 2016); (2) although ASKfm may be similar to one-to-many context of social media, ASKfm users have no idea who are following them. It means that they have no idea to what extent their answer (which may contain their personal information) is published. Here, ASKfm user may have different motives on selfdisclosures and privacy management that have not been explored by scholars.

\section{METHOD}

This research used qualitative method with phenomenological approach to provide understanding of "lived experiences" (van Manen, 1990 as cited in Creswell, 2007, p. 58) of persons who disclosed personal information to wide and unknown publics through ASKfm. Hence, the research targeted ASKfm users in Indonesia, especially in Jakarta and Bekasi region. The participants of this research were between 18 to 24 years old because they were the most common users of ASKfm as reported by Quantcast in 2016.

Six Indonesian ASKfm users (three males and three females) were selected to be participants of this research. They had ASKfm account and actively participated in responding anonymous questions or comments. The selection of participants was based on initial observation of targeted participants' ASKfm account; to confirm whether they had answered anonymous questions at least five times, had minimum 100 "likes" of answers in total, and had revealed their personal information in those answers such as sharing personal opinion, offline activities, photos and videos related to some kinds of information as stated by DeVito (2014, p. 56).

Primary data were collected through in-depth interview with semistructured format. Through this format, specific questions were generated from general questions with a focus on personal information revealed by the participants and their privacy controls in ASKfm. After 
the participants signed research's consent form, general questions were given about their use of ASKfm; their reason to disclose their personal information to unknown public; and their privacy controls in ASKfm. These questions were developed to specific questions prior to interviewing and then the authors decided which issues to pursue (Daymon and Holloway, 2011). All interviews were translated from Indonesian into English language.
Once the data gathered, the authors described the experiences of participants in disclosing their personal information. Relevant data were selected by finding statements in the interviews to be grouped in some keywords (categorization) in relation to the research questions. After that, the authors interpreted the data with the support of secondary data which are literature review of journals, theses, books of theory, and results from the previous researches (Creswell, 2007, p. 159).

\section{RESULT AND DISCUSSION}

\subsection{Participants}

Table 1: Participants' Data

\begin{tabular}{cccccc}
\hline No & Name & Sex & $\begin{array}{c}\text { Age } \\
\text { (y.o) }\end{array}$ & $\begin{array}{c}\text { Number of Likes } \\
\text { (updated Dec 9, } \\
\text { 2016) }\end{array}$ & $\begin{array}{c}\text { Number of } \\
\text { Followers }\end{array}$ \\
\hline 1 & Isty Marta Sukma & Female & 20 & 72,703 & 9,827 \\
\hline 2 & $\begin{array}{c}\text { Harnessia Caroline } \\
\text { Wijaya }\end{array}$ & Female & 20 & 881 & 128 \\
\hline 3 & Nabila Nur Annisa Rawi & Female & 21 & 1,434 & 414 \\
\hline 4 & Angga Febian & Male & 20 & 451 & 111 \\
\hline 5 & Mohammad Ilham Agasi & Male & 21 & 6,511 & 281 \\
\hline 6 & Aswin Atonie & Male & 24 & 338 & 213 \\
\hline & & & & &
\end{tabular}

Participants have signed consent form and fully understand the purpose of their participation in this research.

\subsection{Results}

Through the data collection, there are two participants who tended to have individualistic. Isty usually spent her time mostly for doing activities herself. He did not want to join certain group of friends, but only wanted to have usual friendship. She did not like to be really closed and dependent with her friends. Similar to Isty, Aswin preferred to be alone because he was introvert and did not like crowded place. He thought that by having most of the time for himself, he could develop himself better rather than wasting time with friends for only talking nothing-serious topics. Joining organizations was just a tool for him to show that he could be successful in everything. Moreover, he admitted that he always found solutions of his problems by himself.

This show contrary result toward the previous finding, stating that Indonesia is one of collectivistic country (Samovar, Porter, \& McDaniel, 2009, p. 199-200; Novera, 2004). Related to the finding, Isty and Aswin are the participants who have built relationship and even maintained it after the anonymous ASKfm users revealed their identity. 
"My main purpose is looking for acquaintances, friends. Because through ASKfm, I can have many friends who have broad knowledge and most of them are friendly ... I can know their point of view or mindset before I choose who I want to make friend with. If they have good mindset, I want to make friend with them," Isty said.

"The purpose of ASKfm is not to hold my personal information that I want to share because I think that I have a life story which I can share to other people and it can help them. In this way, my personal information are still in my maintenance," Aswin said.

This shows contradictive finding towards previous finding by Veltri et al. (2011) stating that individualistic societies receive more benefit from self-presentation on Facebook by publishing their life, thoughts, and experiences.

Both male and female participants basically have same motivation in doing self-disclosures in ASKfm. They do selfdisclosure to store their information (tips, opinion, and advices) to help the anonymous ASKfm users, hence got enjoyment (feel happy) from more questions received and indirectly felt that they were quite popular or noticeable in social media and/or real life.

"I'm happy when there are many questions for me although there may be many random or weird questions. Because it means they want to know about me. If the users asked me as the anonym, it means they were shy or there must be something bothers him or her about me. It feels like they are interested in me either in a good or bad way ... I prefer to consider myself as noticeable," Angga said.

They felt happy when they got likes because they thought that their opinion were appreciated or the likers agreed to their opinion, but did not expect it. Some participants promoted their ASKfm account by putting the URL on their Instagram's profile.

Although all participants had no idea about the identity of ASKfm users who asked them, they could surely know that they sometimes had conversation with the same user because of the same writing style and seemed to be continuous questions from previous topic. Here, reciprocal information between participants and anonymous ASKfm users through question-and-answer for several times led them to build relationship, and for some participants they intentionally maintain the relationship through chatting applications.

Participants were were fully aware that their answers will be visible to wider publics once their answer got likes and that other users can share their answer in a form of link to other social media. Even so, they were not afraid to share their personal information because they thought what they shared were still proper and would not cause negative effect that may hurt other people. They concern on their privacy, thus are careful when disclose their personal information by setting their own boundary to what extent they share their personal information in ASKfm. For example, Mohammad did not and will not answer anonymous questions which intended to dig his personal problem or question that may make him to end up with vilifying or mocking or hurting someone. He usually answered with jokes (e.g. GIF pictures, funny pictures from other social media) to questions he thought inappropriate such as asking to PAP of his recently calls. When he felt that the anonymous questions were annoying such asking to PAP his vulgar or nude photo, he would directly delete the questions. Meanwhile, Nabila did not answer to anonymous questions which she thought inappropriate (e.g. asking her family background and parent's job) or 
annoying (e.g. asking whether she ever did making love). It means that there is no difference of motivation and privacy management between male and female participants.

Furthermore, "optimistic bias" attitude was showed by some participants, both collectivists and individualists, who did not read overall contents of Terms of Use, Safety Centre, Community Guidelines, Privacy Policy, and Cookie Policy in ASKfm. Although they were aware of possible risks in disclosing personal information in ASKfm, they believed that they would not get any serious privacy damage because they have filtered questions which they thought appropriate to be answered and to what extent they shared their personal information. Therefore, they did not change their Privacy Settings. Isty who ever changed her Privacy Settings because she did not want to receive a lot of questions at that time, not because of her privacy concern. It means that both collectivistic and individualistic participants have less effective privacy settings in ASKfm. There is also contradictive finding from the previous research in Morocco; it does not show the attitude because Moroccan people were aware of likelihood of privacy violation, thus they preferred to be more passive in disclosing information in Facebook. In this research, participants were actively disclosing their personal information but did not change their Privacy Settings although they were aware of serious possible risks, but never experienced them.

All participants showed similar characteristic of high-context culture. They prefer to talk subtly when expressing their feeling to others and sometimes keep silent to avoid worse problem that may happen. Some participants gave joke answer such as posting funny GIF picture to inappropriate questions or only gave indirect description to anonymous questions for avoiding bad consequences. Moreover, not all of them perceived ASKfm as a tool to increase quality of life. They, show lack of trust in ASKfm because of possible negative effects as stated in Arab Social Media Report (2005, p. 2), for example, Angga who has reduced his intensity to use ASKfm because he thought that ASKfm and other social media are very addicted. Hence, he wanted to achieve something which is more worthy now. Moreover, Isty also turned off "Allow Anonymous Questions" button and even deactivated her account because she felt annoyed to a lot of anonymous questions.

Some contradictive findings can happen because of different context used in each research. Previous researches focused on Facebook and Twitter as the platform to be explored, while this research focuses on ASKfm. Participants were motivated to answer anonymous questions for fun, relieving stress, and fill leisure time when they are bored. Participants also did not take serious concern in sharing personal information in ASKfm. This is because they also used ASKfm as a source of information to see other users' mindset and perception as well as to keep updated to trending topic.

Since ASKfm is one-to-many context of social media, participants were more careful in managing disclosures in ASKfm. However, unlike other social media such Facebook and Twitter, they did not perceive privacy settings as important for them in managing privacy. Instead of changing their Privacy Settings, they follow the default settings of ASKfm although they were aware of possible privacy violation and risks in ASKfm. They only filtered questions they thought appropriate to be answered.

This research critically argued that self-disclosures in ASKfm were merely done by the users for getting enjoyment rather than relationship building and maintenance as happen in other social 
media such Facebook and Twitter. In fact, ASKfm is a Q\&A-style social media in which the ASKfm users who give questions have limited space of 300 words. This research has shown that there is lack of reciprocal information among the users. It has been proved that ASKfm enables its users to get new acquaintances by doing selfdisclosures in temporary relationship to stranger, but not to have continuous relationship. Moreover, ASKfm does not have a feature for private communication, hence in this research, ASKfm users had to move to other private communication platform. This may be also the reason of this research's participants begun to refrain from using ASKfm actively nowadays.

Finally, based on this research, it is suggested for further research to explore the motives of ASKfm users and non-users (users without ASKfm account) to communicate with other users anonymously. Besides, ASKfm may provide new feature for its users which enables them to have private conversation like other social media have, such as Facebook has Facebook Messenger or Twitter has Direct Message feature.

\section{CONCLUSION}

Self-disclosures in ASKfm involved personal evaluation towards rewards and costs received when they answered to anonymous questions. It motivated and determined the participants to what extent they revealed their personal information in answering anonymous questions. Different self-disclosure behavior was not shown in different gender since both male and female participants had similar perception toward personal information to be shared in ASKfm. Although it is believed that Indonesia is one of collectivistic countries, not all its societies are collectivist. Individualists received more benefit on relationship building and maintenance, while collectivists received more benefit on enjoyment. Self-presentation indirectly caused motivation to do self-disclosure since some participants promoted their ASKfm account in other social media to get more questions and they felt quite popular. Participants showed "Optimistic bias" attitude since they perceived Terms of Use and Privacy Policies in ASKfm as unimportant matter; they trusted to ASKfm as a responsible social media which will protect their personal information. Therefore, instead of change Privacy Settings, they preferred to only choose appropriate questions to be answered (filtering questions). 


\section{REFERENCES}

Ahmed, A. A. A. M. (2015). "Sharing is Caring": Online Self-disclosure, Offline Social Support, and Social Network Site Usage in the UAE. Contemporary Review of the Middle East, 2(3), 192-219.

Al-Kandari, A., Melkote, S. R., \& Sharif, A. (2016). Needs and Motives of Instagram Users that Predict Self-disclosure Use: A Case Study of Young Adults in Kuwait. Journal of Creative Communications, 0973258616644808 .

Araújo, C. S., Corrêa, L. P. D., da Silva, A. P. C., Prates, R. O., \& Meira, W. (2014, October). It is not just a picture: revealing some user practices in instagram. In Web Congress (LA-WEB), 2014 9th Latin American (pp. 19-23). IEEE.

Ask.fm traffic and demographic statistics by Quantcast. (n.d.). Accessed on 26 October 2016. Retrieved from https://www.quantcast.com/ask.f $\mathrm{m}$ \#trafficCard on October 26, 2016

Bargh, John. A., McKenna, Katelyn. Y. A., \& Fitzsimons, Grainne. M. (2002). you see the real me? Activation and expression of the "true self on the internet. Journal of Social Issues, 58(1), 33-48.

Bevan-Dye, A. L., \& Akpojivi, U. (2016). South African Generation $Y$ students' self-disclosure on Facebook. South African Journal of Psychology, 46(1), 114-129.

Chen, G. M. (2015). Why do women bloggers use social media? Recreation and information motivations outweigh engagement motivations. new media \& society, 17(1), 24-40.
Christofides, E., Muise, A., \& Desmarais, S. (2009). Information disclosure and control on Facebook: are they two sides of the same coin or two different processes?. CyberPsychology \& Behavior, 12(3), 341-345.

Christofides, E., Muise, A., \& Desmarais, S. (2012). Hey mom, what's on your Facebook? Comparing Facebook disclosure and privacy in adolescents and adults. Social Psychological and Personality Science, 3(1), 48-54.

Creswell, J. W. (2007). Qualitative Inquiry\& Research Design . Thousands Oaks.

DeVito, J. A. (2014). Human communication: The basic course. Pearson Higher Inc. pg. 56-60.

Ellison, N. B., Blackwell, L., Lampe, C., \& Trieu, P. (2016). "The Question Exists, but You Don't Exist With It": Strategic Anonymity in the Social Lives of Adolescents. Social Media+ Society, 2(4), 2056305116670673 .

Golbeck, J. (2015). Introduction to Social Media Investigation: A Hands-on Approach. Syngress.

Hosseinmardi, H., Han, R., Lv, Q., Mishra, S., \& Ghasemianlangroodi, A. (2014). Analyzing negative user behavior in a semi-anonymous social network. CoRR abs, 1404.

Hosseinmardi, H., Mattson, S. A., Rafiq, R. I., Han, R., Lv, Q., \& Mishra, S. (2015). Detection of cyberbullying incidents on the instagram social network. arXiv preprint arXiv:1503.03909. 
Humphreys, L., Gill, P., \& Krishnamurthy, B. (2014). Twitter: a content analysis of personal information. Information, Communication \& Society, 17(7), 843-857.

Joinson, A. N. (2001). Self-disclosure in computer-mediated communication: The role of selfawareness and visual anonymity. European journal of social psychology, 31(2), 177-192.

Kang, R., Brown, S., \& Kiesler, S. (2013, April). Why do people seek anonymity on the internet?: informing policy and design. In Proceedings of the SIGCHI Conference on Human Factors in Computing Systems (pp. 26572666). ACM.

Krasnova, H., Kolesnikova, E., \& Guenther, O. (2009). "It Won't Happen To Me!": Self-Disclosure in Online Social Networks. Amcis 2009 Proceedings, 343.

Lai, C. Y., \& Yang, H. L. (2015). Determinants of individuals' selfdisclosure and instant information sharing behavior in micro-blogging. new media \& society, 17(9), 1454-1472.

Li, H. H. S., Yang, Z., Lv, Q., Han, R. I. R. R., \& Mishra, S. (2014, December). A comparison of common users across instagram and ask. fm to better understand cyberbullying. In Big Data and Cloud Computing (BdCloud), 2014 IEEE Fourth International Conference on (pp. 355-362). IEEE.

Liang, H., Shen, F., \& Fu, K. W. (2016). Privacy protection and selfdisclosure across societies: A study of global Twitter users. New Media \& Society, 1461444816642210.
Madden, M. (2012). Privacy management on social media sites. Pew Internet Report, 1-20.

Masur, P. K., \& Scharkow, M. (2016). Disclosure management on social network sites: Individual privacy perceptions and user-directed privacy strategies. Social Media+ Society, 2(1), 2056305116634368.

McKenna, Katelyn. Y. A., Green, Amie. S., \& Gleason, Marci. E. J. (2002). Relationship formation on the internet: What's the big attraction? Journal of Social Issues, 58(1), 9-31.

Mulyana, D. (2008). Ilmu komunikasi: Suatu pengantar. Bandung: Remaja Rosdakarya.

Novera, I. A. (2004). Indonesian Postgraduate Students Studying in Australia: An Examination of Their Academic, Social and Cultural Experiences. International Education Journal, 5(4), 475-487.

Rains, S. A., \& Brunner, S. R. (2015). The Outcomes of Broadcasting SelfDisclosure Using New Communication Technologies Responses to Disclosure Vary Across One's Social Network. Communication Research, 0093650215598836.

Samovar, L. A., Porter, R. E., McDaniel. (2009). Communication between cultures. Cengage Learning.

Sheldon, P. (2015). Social media: Principles and applications. Lexington Books.

Tian, Q. (2013). Social anxiety, motivation, self-disclosure, and computermediated friendship $A$ path analysis of the social interaction in the blogosphere. Communication Research, 40(2), 237-260. 
Pheseline Felim, Dindin Dimyati \& Mohammad Shihab, $\mathrm{Kfm}$ : Motives of Self-Disclosure to Anonymous Questions

Trepte, S. (2015). Social Media, Privacy, and Self-Disclosure: The Turbulence Caused by Social Media's Affordances. Social Media+ Society, 1(1), 2056305115578681.

Tsay-Vogel, M., Shanahan, J., \& Signorielli, N. (2016). Social media cultivating perceptions of privacy: A 5-year analysis of privacy attitudes and selfdisclosure behaviors among Facebook users. new media \& society, 1461444816660731.

Veltri, N. F., Krasnova, H., \& Elgarah, W. (2011). Online Disclosure and Privacy Concerns: a Study of Moroccan and American Facebook Users. In AMCIS.
Waters, S., \& Ackerman, J. (2011). Exploring privacy management on Facebook: Motivations and perceived consequences of voluntary disclosure. Journal of Computer-Mediated Communication, 17(1), 101-115.

West, R. L., \& Turner, L. H. (2006). Introducing communication theory: Analysis and application. McGraw-Hill Humanities/Social Sciences/Languages. 
Jurnal komunikasi, Volume 13, Nomor 1, Oktober 2018 